\title{
SYNAPTIC FACILITATORY AND DEPRESSANT ACTIONS OF 3,4-DIAMINOPYRIDINE: CORRELATION WITH ANTICURARE PROPERTIES
}

\author{
William K. RIKER, Brian R. APATOFF* and Kazuhiko SASAKI ${ }^{\dagger}$ \\ Department of Pharmacology, School of Medicine. The Oregon Health Sciences University. \\ Portland. OR 97201. U.S.A. \\ *University of Chicago, School of Medicine, Chicago, IL 60637, U.S.A. \\ † Department of Physiology, Iwate Medical University. School of Medicine, Iwate 020, Japan
}

Accepted July 1, 1983

\begin{abstract}
This study aimed to define the complete concentration-effect relationship for anticurare effects of 3,4-diaminopyridine (3,4-DAP) in the isolated sympathetic ganglion of the bullfrog. Synaptic transmission was monitored by extracellular and intracellular recordings of the postganglionic response to preganglionic stimulation. A previous study showed that in the bulifrog sympathetic ganglion 3.4-DAP caused stimulus-bound repetitive postganglionic responses (SBR) to each single preganglionic stimulus. The concentration-effect relationship for 3,4-DAP-induced SBR was bell-shaped, and the descending limb of the curve reflected progressive suppression of SBR while normal synaptic transmission was maintained. In the present study a detalled concentrationeffect analysis of 3,4-DAP's anticurare action also resulted in a bell-shaped curve nearly congruent with that for SBR. SBR and anticurare effects of 3,4-DAP therefore occupy a common concentration-effect domain, and this suggests that a common mechanism (increased transmitter release) may account for both effects.
\end{abstract}

The aminopyridines augment synaptic transmission in a variety of peripheral and central synapses, apparently by an action on prejunctional nerve terminals to increase transmitter release (1). Amplitudes of postjunctional potentials are therefore enhanced $(2,3)$, and repetitive postjunctional responses to a single prejunctional stimulus can also be generated (4, 5). Not unexpectedly, the aminopyridines exhibit anticurare efficacy in animals (1-7), and man (8-10) and have antimyasthenic properties as well (11)

In a recent study of 3,4-diaminopyridine (3,4-DAP) in bullfrog sympathetic ganglion we found that its augmentative, anticurare, and depressant actions on synaptic transmission occurred throughout a concentration range greater than three orders of magnitude (12). The input-output relation- ship in the bullfrog synapse, normally $1: 1$. was altered by 3,4-DAP so that each single preganglionic stimulus elicited a brief. synchronous burst of repetitive postganglionic responses. We have reported this type of drug-induced stimulus-bound repetitive firing (SBR) previously for neostigmine and congeners $(13,14), \mathrm{Cs}^{+}(15)$, ethanol (16), and thiazides (17)

The anticurare action of 3,4-DAP in the bullfrog ganglion caused the concentrationeffect curve for $d$-tubocurarine $(d-T c)$ transmission block to be shifted approx. fourfold to the right (12). This anticurare effect has now been subjected to more detailed analysis. and the present report shows that both 3,4DAP-induced repetitive firing (SBR) and the anticurare action are characterized by bellshaped concentration-effect curves that are 
nearly congruent. Thus, the SBR and the anticurare effect of 3.4-DAP may be viewed as different manifestations of a common mechanism; most likely increased transmitter release consequent to a primary presynaptic site of drug action.

\section{Materials and Methods}

The experiments were performed in vitro on sympathetic ganglia isolated from Rana catesbiana (300-500 g) of either sex. The eighth or ninth ganglion in the paravertebral chain, together with its attached preganglionic trunk and postganglionic ramus entering the corresponding spinal nerve, was excised and adherent connective tissue was removed under microscopic control.

Extracellular recordings of the transmitted postganglionic compound action potential (CAP) in response to preganglionic stimulation were obtained from a pair of platinumiridium electrodes, one on the caudal pole of the ganglion and the other on the spinal nerve containing the postganglionic fibers. Supramaximal stimulation (duration, 0.2 $\mathrm{msec}$ ) of the preganglionic trunk was delivered at a frequency of $0.1 \mathrm{~Hz}$ unless indicated otherwise. The preparation was submerged under paraffin oil in a special chamber allowing selective immersion of the ganglion in any one of multiple wells containing Ringer's solution or drug solutions of known concentration (12).

For intracellular recording the ganglion was fixed on a Sylgard floor in a central perfusion compartment of a plexiglass chamber. The attached ramus-spinal nerve and the preganglionic trunk were drawn into separate compartments lateral to the central perfusion compartment, covered with vaseline, and stimulating electrodes were applied. Thus, preganglionic (orthodromic) and/or postganglionic (antidromic) stimuli could be delivered. The ganglion was illuminated with darkfield optics under 50-100* magnification for direct visualization of individual neurons and placement of the microelectrode tip.

Capillary microelectrodes with resistances between 20-40 M 2 were filled with $1.5 \mathrm{M}$ potassium citrate or $3 \mathrm{M} \mathrm{KCl}$ and connected to the input of a unity-gain preamplifier with negative capacitance compensation (Dagan 8500). Signals from intracellular or extracellular electrodes were amplified, displayed on an oscilloscope and photographed or recorded on magnetic tape for later analysis.

The composition of the Ringer's solutions was as follows ( $\mathrm{mM}$ ): $\mathrm{NaCl}$ (112): $\mathrm{KCl}(2.0)$; $\mathrm{CaCl}_{2}$ (1.8); HEPES Buffer (5.0); NaOH (1.8). With concentrations of 3,4-diaminopyridine greater than $1 \mathrm{mM}$, the $\mathrm{pH}$ of the Ringer's solution was maintained at 7.2 by reducing the concentration of $\mathrm{NaOH}$. The following drugs were used: 3,4-diaminopyridine (Aldrich): d-tubocurarine chloride (Calbiochem).

\section{Results}

Repetitive postganglionic firing in 3,4diaminopyridine (3,4-DAP): With intracellular recording in drug-free normal Ringer's solution, a single preganglionic (orthodromic) stimulus evoked a single, all-or-none synaptic potential-spike response in individual postganglionic $B$ neurons, as shown in Fig. 1A. This input-output characteristic in bullfrog sympathetic ganglion cells is attributable to the predominant 1:1 preganglionic: postganglionic innervation ratio. After addition of 3,4-DAP, however, each single orthodromic stimulus elicited a brief repetitive burst in which one, sometimes two. additional action potentials occurred quickly after the initial spike (Fig. 1B). In contrast, antidromically evoked responses in the presence of 3,4-DAP never displayed repetitive firing (Fig. $1 \mathrm{~A}, \mathrm{~B}$ ).

For any given cell, the peak-to-peak interval between primary and repetitive 

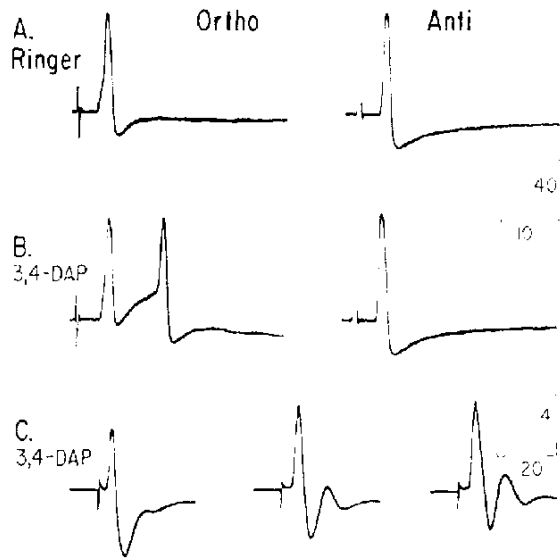

Fig. 1. Intracellular (A, B) and extracelluiar (C) recordings in bullfrog sympathetic ganglion. A. Control responses to preganglionic (Ortho) and postganglionic (Anti) stimulation, at $0.1 \mathrm{~Hz}$, in a single ganglion cell. B. Same cell after 6 min perfusion with $200 \mu \mathrm{M} 3$ 3-diaminopyridine (3,4-DAP). Note that the single Ortho stimulus now evokes two spikes (SBR, see text) whereas Anti response is unchanged. C. Extracellular recordings of postganglionic compound action potential (CAP) responses to single supramaximal preganglionic stimuli $(0.1 \mathrm{~Hz})$ showing 3.4-DAP-induced SBR. Left to right, recordings in 2.20, and $200 \mu \mathrm{M} 3.4$-DAP. At 20 and $200 \mu \mathrm{M} 3.4-$ DAP note the second, smaller CAP (SBR), compared to the threshold emergence of the repetitive $\mathrm{CAP}$ at $2 \mu \mathrm{M}$. Calibrations in $\mathrm{mV}$ and msec.

spike(s) remained constant, and the range of interspike intervals in more than seventy cells impaled varied from 8 to $15 \mathrm{msec}$. This range of interspike intervals for 3,4-DAPinduced stimulus-bound repetitive firing (SBR) is also characteristic of the SBR generated in bullfrog sympathetic ganglion by neostigmine and other drugs (12-17).

Figure $1 \mathrm{C}$ shows that 3.4-DAP-induced SBR could also be monitored by extracellular recording of the postganglionic compound action potential (CAP) response to supramaximal stimulation $(0.1 \mathrm{~Hz})$ of the preganglionic trunk. Under these conditions SBR is manifested by a second (repetitive) CAP arising from the positive phase of the initial CAP (Fig. 1C). Because the amplitude of the extracellularly recorded SBR is pro- portional to the percentage of cells with $\mathrm{SBR}$, this convenient recording method was employed in our previous study (12) to determine the concentration-effect (incidence) relationship for 3,4-DAP-induced SBR. That relationship was a bell-shaped curve spanning a concentration range greater than 1.000-fold (12), and it has been reproduced, with several additional points, in Fig. 3. Thus, increases in 3,4-DAP concentration beyond those causing maximal SBR amplitude resulted in a selective, gradual suppression and abolition of SBR, without any reduction in the amplitude of the primary CAP.

Block of 3,4-DAP-induced SBR, and of transmission, by d-tubocurarine ( $d-T c)$ : 3.4DAP-induced SBR could also be selectively suppressed and abolished by $d-T c$ concentrations below those that caused transmission block. Figure 2 shows the $d-T c$ concentration-effect relationship for suppression of SBR produced by $30 \mu \mathrm{M}$ (open squares) or $200 \mu \mathrm{M}$ (open triangles) 3.4-DAP. In these experiments the ganglia were first exposed to 3,4-DAP for 30 to $50 \mathrm{~min}$, sufficient time to produce SBR with stable, constant amplitude.

The ganglia were then incubated, sequentially. in solutions containing progressively higher concentrations of $d-T c$ plus the original 3.4-DAP concentration. Reductions in the amplitude of SBR were then measured, relative to the control amplitude in 3,4-DAP alone, and plotted.

To the right of the SBR suppression curves in Fig. 2 is the concentration-effect relationship (open circles) for transmission block (reduction of primary CAP amplitude) by d-Tc in Ringer's solution without 3,4-DAP. The remaining two curves in Fig. 2 also show that the $d-T_{c}$ transmission-blocking curve was shifted to the right approx. fourfold when the $\mathrm{d}$-Tc blocking bioassay was carried out in the presence of $30 \mu \mathrm{M}$ (crosses) or $200 \mu \mathrm{M}$ (closed circles) 3,4-DAP. While these latter 


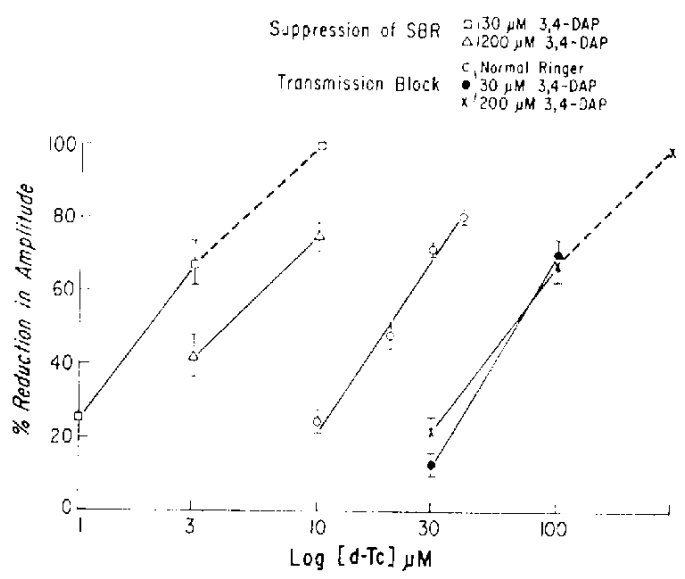

Fig. 2. Log concentration-effect relationships for d-tubocurarine (d-Tc) suppression of 3,4-DAP. induced SBR and for transmission block in the builfrog sympathetic ganglion. Ordinate: Percent reduction in amplitude, of SBR and of the primary transmitted postganglionic CAP (transmission block). Abscissa: Log d-Tc concentration. The two curves at the left show d-Tc suppression of SBR induced by $\left.30\left(L^{-}\right]\right)$or $200(\Delta) \mu \mathrm{M} 3,4$-DAP. The middle curve shows transmission block by $d$-Tc in normal Ringer (O) and the two curves at right are for $\mathrm{d}$-Tc transmission block in the presence of 30 (O) and 200 (X) $\mu \mathrm{M} 3,4$-DAP. Each point is the $\bar{X} \pm$ S.E.M. of 7-11 experiments, except for the $10 \mu \mathrm{M}$ d-Tc vs. $30 \mu \mathrm{M}$ 3.4-DAP point, where $n=5$.

two curves demonstrate the anticurare action of 3.4-DAP they are also the appropriate reference point for comparison with the two curves at the extreme left in Fig. 2, showing d-Tc suppression of 3,4-DAP-induced SBR. Hence, the suppression of SBR by $d-T c$ could be dissociated from its transmission blocking action with remarkable concentration selectivity (12).

Since 30 and $200 \mu \mathrm{M}$ 3,4-DAP each produced the identical four-fold rightward shift in the d-Tc transmission-blocking curve (Fig. 2), we assumed this to be a maximum anticurare action. It is reasonable to expect that the rightward shift of the $\mathrm{d}$-Tc blocking curve occurs in graded steps at 3,4-DAP concentrations below $30 \mu \mathrm{M}$. Considering that aminopyridines are strong bases it would also be expected that 3,4-DAP

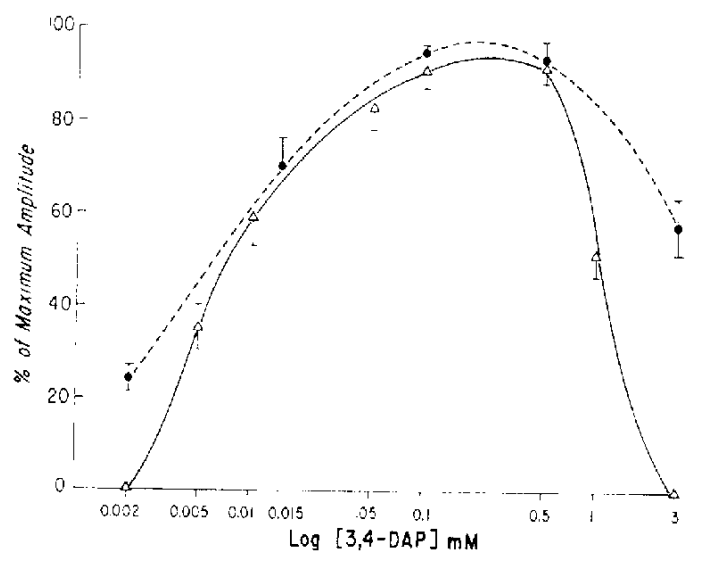

Fig. 3. Log 3,4-DAP concentration-effect relationship for anticurare action ( ...... in bullfrog sympathetic ganglion, combined with the concentration-effect for 3,4-DAP-induced SBR $(\triangle-\triangle)$ The SBR curve is derived from a previous study (12). Ordinate: Percent of maximum amplitude of SBR (see Fig. 2 legend) and of reversal of d-Tc transmission block (see text). Abscissa: Log 3.4-DAP concentration, in $\mathrm{mM}$. Each point in the anticurare curve is the $\bar{x} \pm$ S.E.M. of 8 experiments. Note that both the SBR and anticurare concentration-effect relationships are bell-shaped and nearly congruent.

concentrations above $200 \mu \mathrm{M}$ might nullify their own anticurare effect by simultaneously depressing transmission, again in graded fashion. The complete concentrationanticurare spectrum of 3,4-DAP would thus be a bell-shaped curve if these expectations are correct. The succeeding section describes the procedure used to test this premise.

\section{3,4-DAP anticurare action. Concentration-} effect characteristic: Extracellular recordings of the postganglionic CAP response to supramaximal preganglionic stimulation $(0.1$ $\mathrm{Hz}$ ) were made in 8 ganglia. After obtaining control records in drug-free normal Ringer. ganglia were then bathed in $30 \mu \mathrm{M} d-T_{c}$ for 30 to $40 \mathrm{~min}$ to achieve stable transmission block. d-Tc. $30 \mu \mathrm{M}$, reduced the CAP amplitude by $8.31 \pm 3.0 \%$ ( $x \pm$ S.E.M., $N=8$ ). The ganglia were then bathed in a succession of 3,4-DAP solutions (each solution also containing $30 \mu \mathrm{M} \mathrm{d}-\mathrm{Tc}$ ) of ascending con- 
centration: $2,15,100,500 \mu \mathrm{M}$ and $3 \mathrm{mM}$. Incubation time in each solution was $45 \mathrm{~min}$. sufficient to attain the peak anticurare effect for any given 3.4-DAP concentration.

Maximum reversal of $\mathrm{d}-\mathrm{Tc}$ block occurred in three ganglia at $100 \mu \mathrm{M}(\mathrm{x}=88 \%$ of Ringer control CAP amplitude), and in 5 at $500 \mu \mathrm{M}$ 3.4-DAP ( $x=118 \%$ of Ringer control CAP amplitude). For data analysis the maximum reversal amplitude of the CAP in each ganglion was assigned the value of $100 \%$ and the CAP amplitudes at all of the other 3,4DAP concentrations then expressed as a percentage of the maximum.

The 3,4-DAP anticurare data are plotted in Fig. 3 together with reproduction of the bell-shaped curve for 3,4-DAP production of SBR. It is evident that the two concentrationeffect relationships are both bell-shaped and nearly congruent, the greatest departure from congruency being at the $3 \mathrm{mM}$ 3.4-DAP level.

\section{Discussion}

There has now beer! repeated confirmation that aminopyridines, notably 4-aminopyridine (4-AP) and 3,4-diaminopyridine (3,4-DAP), enhance th amplitudes of postjunctional potentials. In a recent review, Thesleff (1) has summarized the facilitatory actions of aminopyridines at many kinds of synapses, as well as the evidence that points to a primary, direct action of these drugs on prejunctional nerve terminals to increase transmitter release. The well-documented anticurare effect of aminopyridines at neuromuscular junctions $(3-10,18)$ is reasonably based on this increased transmitter release, with consequent augmentation of endplate potential amplitude and, especially, rate of rise (18)

Aminopyridines also enable single prejunctional stimuli to evoke repetitive postjunctional responses at frog $(4,5,19)$, and rat (4) neuromuscular junction. In a previous report (12) and in the present one, we have shown that 3,4-DAP also caused stimulusbound repetitive postganglionic responses (SBR) to each single preganglionic stimulus in bullfrog sympathetic ganglion. The complete concentration-effect analysis of 3,4DAP-induced SBR (Fig. 3) is noteworthy for the bell-shaped relationship spanning a concentration range more than 1,000-fold. This characteristic is not unique to 3,4-DAP. for we have previously reported bell-shaped concentration-effect curves with equally extensive concentration ranges, for SBR induced by neostigmine and congeners in bullfrog sympathetic ganglion $(13,14)$.

The descending limbs of the bell-shaped curves for 3.4-DAP, or neostigmine-induced, SBR reflect the dual facilitatory and depressant properties intrinsic to each of these drugs. Since the descending limb in Fig. 3 represents a selective depression of SBR (i.e.. at 3,4-DAP concentrations below those that block transmission) it also discloses that a synaptic depressant effect of 3,4-DAP is manifest even before transmission block is evident. At the neuromuscular junction the actions of neostigmine and related anticurare drugs have also long been known to include depression at higher doses, in animal studies $(20,21)$ and in clinical reversal of curariform neuromuscular block $(22,23)$.

Despite an extensive literature concerning aminopyridines, relatively little attention has been paid to the junctional depressant properties of these drugs. The present study shows that the combined synaptic facilitatory and depressant properties of 3,4-DAP apply not only to SBR, but to the anticurare effects as well. Our analysis of the reversal of $d-T c$ transmission block by 3,4-DAP demonstrates that the anticurare effect is also described by a bell-shaped concentration-effect relationship, one essentially congruent with that for SBR (Fig. 3). Congruency of the 3,4-DAP SBR and anticurare curves does not imply that SBR was responsible for the anticurare 
effect. To the contrary, there was no SBR at any point in the reversal of $d-T c$ block by 3,4-DAP because of the simultaneous presence of $30 \mu \mathrm{M} \mathrm{d}-T_{c}$ in the bath (Figure 2 shows that concentrations of $d-T_{c}$ much less than $30 \mu \mathrm{M}$ selectively abolished SBR). Rather, the congruent $S B R$ and anticurare curves simply establish that two different minifestations of synaptic facilitation and depression by 3,4-DAP have a common concentrationeffect domain and, therefore, probably a common mechanism of action. The departure from near-perfect-congruency of the SBR and anticurare curves at the higher, 3,4-DAP concentrations (Fig. 3) may be attributable in part to the experimental procedure, i.e., to the cumulative method of concentrationeffect analysis. Thus, it is likely that the lower, maximally effective anticurare concentrations of 3,4-DAP not only raised $d-T c-$ depressed synaptic potentials to spike threshold, but also augmented the amplitudes well above threshold at a large fraction of junctions. Also, since 3,4-DAP-induced SBR is much more sensitive to depression by $\mathrm{d}-$ Tc and TEA than is transmission (12), it is not surprising that the depressant effects of higher 3,4-DAP concentrations suppress SBR more readily than they do transmission (Fig. 3).

Durant and colleagues (24) demonstrated that 4-AP is a potent antagonist of $d-T_{c}$ and hexamethonium blockade in the superior cervical ganglion of the cat. The present study of 3,4-DAP in bullfrog sympathetic ganglion supports and extends their findings; particularly in providing the first complete concentration-effect analysis of aminopyridine anticurare action. Our data may also be relevant to aminopyridine anticurare action at the mammalian neuromuscular junction. Miller and his colleagues $(8,25)$ have shown that 4 -aminopyridine acts synergistically with neostigmine or pyridostigmine to antagonize pancuronium neuromuscular paralysis in man and Org NC 45 paralysis in rat. Among the advantages of combining 4-AP with smaller doses of neostigmine would be the reduced requirement for atropine, as well as the efficacy of 4-AP in reversing aminoglycoside block of neuromuscular junctions (9). 3,4DAP would also act synergistically with neostigmine or pyridostigmine to reverse curariform neuromuscular paralysis, and it antagonizes Kanamycin paralysis (26). Considering the extensive 3.4-DAP concentration range encompassed in the bellshaped anticurare and SBR curves (Fig. 3), it is possible that 3,4-DAP might have a greater safety margin than 4-AP with respect to clinical reversal of neuromuscular paralysis. That, and the fact that 3,4-DAP is more potent and much less convulsant than 4-AP (6), suggest that the anticurare interaction of 3,4-DAP and neostigmine may merit clinical investigation.

Acknowledgement: This study was conducted at the Oregon Health Sciences University, and was supported, in part, by research grants from NINCDS (NS-12813) and the Medical Research Foundation of Oregon.

\section{References}

1) Thesleff, S.: Aminopyridines and synaptic transmission. Neuroscience 5, 1413-1419 (1980)

2) Molgó, J., Lemeignan, M. and Lechat, P.: Analysis of the action of 4-aminopyridine during repetitive stimulation at the neuromuscular junction. Eur. J. Pharmacol. 53, 307-311 (1979)

3) Molgó, J., Lemeignan, M. and Lechat, P.: Effects of 4-aminopyridine at the frog neuromuscular junction. J. Pharmacol. Exp. Ther. 203, 653-663 (1977)

4) Lundh, H.: Effects of 4-aminopyridine on neuromuscular transmission. Brain Res. 153, 307-318 (1978)

5) Horn, A.S., Lambert, J.J. and Marshall, I.G.: A comparision of the facilitatory actions of 4 . aminopyridine methiodide and 4-aminopyridine on neuromuscular transmission. $\mathrm{Br}$. J. Pharmacol. 65, 53-62 (1979) 
6) Harvey, A.L. and Marshall, I.G.: The actions of three diaminopyridines on the chick biveriter cervicis muscle. Eur. J. Pharmacol. 44, 303-309 (1977)

7) Bowman, W.C., Harvey, A.L. and Marshalf, I.G.: The actions of aminopyridines on avian muscle. Naunyn Schmiedebergs Arch. Pharmacol. 297, 99-103 (1977)

8) Miller, R.D., Booij, L.H.D.J., Agoston, S. and Crul, J.F.: 4-Aminopyridine potentiates neostigmine and pyridostigmine in man. Anesthesiology 50, 416-420 (1979)

9) Booij, L.H.D.J., Miller, R.D. and Crul, J.F.: Neostigmine and 4-aminopyridine antagonism of lincomycin-pancuronium neuromuscular blockade in man. Anesth. Analg. (Cleve.) 57. 316-321 (1978)

10) Paskov, D.S., Staenov, E.A. and Mitsov, V.V.: New anticurare and analeptic drug pimadin and its use in anesthesia. Exp. Chir. Anesth, 4, 4852 (1973) (in Russian)

11) Lundh, H., Nilsson, $\mathrm{O}$. and Rosen, 1.: Effects of 4-aminopyridine in myasthenia gravis. J. Neurol. Neurosurg. Psychiatry 42, 171-175 (1979)

12) Apatoff, B.R, and Riker, W.K.: The actions of 3.4-diaminopyridine in bullfrog sympathetic ganglia. Brain Res. 252, 277-286 (1982)

13) Riker, W.K. and Guerrero, S.: The production of stimulus-bound repetition by neostigmine in sympathetic ganglion cells. J. Pharmacol. Exp. Ther. 163, 54-63 (1968)

14) Riker, W.K. and Kosay, S.: Drug induction and suppression of stimulus-bound repetition in sympathetic ganglia. J. Pharmacol. Exp. Ther. 173, 284-292 (1970)

15) Riker, W.K., Russell, N.J. and Stolc, S.: Cesium Post-junctional repetitive firing in sympathetic ganglion. Life Sci. 13, 1069-1075 (1973)

16) Montoya, G.A., Riker, W.K. and Russell, N.J. Stimulus-bound repetitive synaptic firing caused by ethanol in smypathetic ganglion. J. Phar- macol. Exp. Ther. 200, 320-327 (1977)

17) Montoya, G., Russell, N.J. and Riker, W.K.: Facilitation of synaptic transmission by thiazides and congeners. Proc. West. Pharmacol. Soc. $18,212-216$ (1975)

18) Jacobs, R.S. and Burley, E.S.: Nerve terminal facilitatory action of 4-aminopyridine: An analysis of the rising phase of the endplate potential. Neuropharmacology 17, 439-444 (1978)

19) Marshall, I.G., Lambert, J.J. and Durant, N.N.: Inhibition of aminopyridine-induced contractile activity in skeletal muscle by tetrodotoxin and by magensium. Eur. J. Pharmacol. 54, 9-14 (1979)

20) Eccles, J.C. and MacFarlane, W.V.: Actions of anti-cholinesterases on endplate potential of frog muscle. J. Neurophysiol. 12, 59-80 (1949)

21) Wescoe, W.C. and Riker, W.F., Jr.: The pharmacology of anti-curare agents. Ann. N.Y. Acad. Sci. 54, 438-455 (1951)

22) Wescott, D.A. and Bendixen, H.H.: A study of neostigmine as a curare antagonist. Anesthesiology 22, 148-149 (1961)

23) Churchill-Davidson, H.C. and Christie, T.H.: The diagnosis of neuromuscular block in man. Br. J. Anaesth. 31, 290-301 (1959)

24) Durant, N.N., Lee, C. and Katz, R.L.: 4-aminopyridine reversal of sympathetic ganglionic blockade in the anesthetized cat. Anesthesiology 52, 381-384 (1980)

25) Booij, L.H.D.J., Van der Pol, F., Crul, J.F. and Miller, R.D.: Antagonism of Org NC 45 neuromuscular blockade by neostigmine, pyridostigmine, and 4-aminopyridine. Anaesth. Analg. 59, 31-34 (1980)

26) Molgó, J., Lemeignan, M., Uchiyama, T. and Lechat, P.: Inhibitory effect of kanamycin on evoked transmitter release. Reversal by 3,4 diaminopyridine. Eur. J. Pharmacol. 57, 93-97 (1979) 